\title{
Planning and Scheduling Process for a Grid-Connected Microgrid Based on Renewable Energy Sources by a Novel Fuzzy Method
}

\author{
Zhao Lijun $\mathbb{D}^{1},{ }^{1}$ Li Qingsheng $\mathbb{D}^{2},{ }^{2}$ and Ding Guanhua $\mathbb{D}^{3}$ \\ ${ }^{1}$ Electrical and Electronic Engineering Department, Chengde Petroleum College, Chengde, Hebei 067000, China \\ ${ }^{2}$ Security Division, Chengde Petroleum College, Chengde, Hebei 067000, China \\ ${ }^{3}$ School of Electronic and Information Engineering, Beihang University, Beijing 100191, China
}

Correspondence should be addressed to Li Qingsheng; li.qingsheng98@gmail.com

Received 17 August 2020; Revised 19 October 2020; Accepted 19 November 2020; Published 5 November 2021

Academic Editor: Mohammad Hassan Khooban

Copyright ( $\odot 2021$ Zhao Lijun et al. This is an open access article distributed under the Creative Commons Attribution License, which permits unrestricted use, distribution, and reproduction in any medium, provided the original work is properly cited.

High growth of the population and developed industries, on the one hand, increased the demanded energy considerably. Growth of the fossil fuel-based power generation, on the other hand, has high environmental effects. Accordingly, renewable energy sources are mainly interested in the recent years as clean resources. These sources also can be economical cases in long term. Nevertheless, their intermittent feature is a main drawback. Integration of these resources with the rest of the grid can increase their advantages and tackle their main drawbacks. In the present work, a novel programming method is proposed for energy management and optimization of operational expenses over a grid-connected microgrid (MG). The considered MG contains various renewable resources. Some uncertainties should be considered in the integration of renewable resources with the grid, which can affect the system performance. Main uncertain parameters are studied in this paper such as predicted values for green power sources, maximum capacity for fuel cells (FCs), energy storage sources (ESSs), maximum capacity for transmission line, and also hourly loads, achieving a higher efficiency. This paper utilized fuzzy idea for presentation of mentioned uncertain parameters in the MG. Moreover, this work employed a 3-stage optimization algorithm for obtaining the best programming for the MG by considering the uncertain parameters. This paper also used a practical test system to validate the proposed method through minimizing the total cost. Additionally, a sensitivity analysis is performed to study the effects of oscillations of all components in the MG and their associated uncertainties over the obtained results.

\section{Introduction}

In electrical networks, restructuring and deregulating presents a proper condition to move from centralized networks to the distributed networks. Centralized power generation results in some problems including power efficiency, security problem, and higher electricity loss, and also such networks include higher expenses in development of transmission/distribution networks chiefly in case of the mountains and low-density regions that are far from the major areas [1]. These stated problems along with the increasing interest for utilization of RES (renewable energy resource) caused improvement in the DGs (distributed generations). These DGs permit to decentralize the energy production [2]. Many of the RESs like microturbines, FCs,
WTs, PV panels, and ESSs can be employed as DGs. Also, an MG is a collection of distributed generations and loads. The daily operation in an MG includes obtaining the lowest expenditure dispatch for distributed generations minimizing the entire operational expenditure to satisfy the demanded power under different limitations including technical, environmental, and operational ones. A microgrid should disconnect itself from the main grid and operate in the form of an autonomous segment with high performance and low emitted pollutions [3]. As well, an MG in a grid-connected situation is able to operate in the form of a sole generatorload entity and exchange power with the power market.

Scheduling of distributed generation in an optimal manner is an important problem that is broadly discussed by researchers. In this work, two main groups are considered 
for various scheduling methods including traditional and fuzzy-based methods. In traditional methods, factors of the scheduling models should be appropriately and precisely presented. Nevertheless, this consideration is not true in practical situations mostly, and their values are selected by experts. It is obvious that the experts' knowledge or prediction outcomes are not adequately exact. The fuzzy concept planned to supply the explanation of measured uncertain parameters of practical stochastic events and presented strong equipment to cope with inexact data. The authors in [4] suggested the decision-making idea in fuzzy systems, which is used by many investigators to solve the fuzzy scheduling issues [4-7]. Next, a number of the traditional and fuzzy-based methods are presented that are utilized for optimum programming of distributed generations.

The authors in [8] proposed an mixed-integer linear programming (MILP) method for optimal programming of an MG containing green resources in an autonomous condition. Zhao et al. [9] presented a mixed-integer quadratic programming method to obtain the best programming for each week for green power sources of an $\mathrm{MG}$, where the lost active energy of distribution system and constraints of distribution lines are regarded. In the considered networks of $[8,9]$, some green resources including the WTs, PV systems, batteries, and FCs are considered, but these papers did not study the uncertainties. In [10], a nonprobabilistic information gap method is employed to handle the uncertainty problem in the suggested virtual energy hub (VEH) system. The authors proposed a hierarchical energy management system in [11] to maximize the total financial profit. They applied the proposed method over multiple home energy hubs in the neighborhood grid and could shave the peak of upper grid. An integrated gas-power network is studied in [12] considering new flexible energy resources. They suggested a multiobjective 2-stage stochastic unit commitment, in which P2G technology is used to increase the wind power dispatch in the power system. Also, they employed demand response program to reduce the total operating cost through shifting the load from peak times to off-peak times. In [13], a smart transactive energy framework is given. They aimed to enhance the financial profit of the whole system. They also presented a comprehensive simulation study for depicting the high efficiency of the suggested method. A multiobjective optimization algorithm is proposed in [14] to optimize the considered system from technical, economic, and environmental aspects. They performed this optimization through scheduling the electric vehicle charging/discharging. They could decrease the energy cost, battery degradation, $\mathrm{CO}_{2}$ emissions, and grid utilization about $88.2,67.0,34.0$, and $90.0 \%$, respectively. In addition, a power system containing a natural gas-based system and wind turbine is studied in [15], where three solutions are suggested to tackle the related problems to the gas system limitations and uncertainties of the wind power. They proposed an IGDT-based robust security-constrained unit commitment model and depicted the impact of emerging flexible sources over a decrement of the total operating cost, as well as the uncertainty of the forecasted wind power. Cau et al. [16] presented a stochastic method over an MG containing green power sources, ESSs, and a hydrogen storage tank. In this reference, the scenario tree model is utilized for insertion of the uncertainties in the suggested approach. Also, Deissenroth et al. [3] proposed a nonlinear method for obtaining of the optimum programming for an $\mathrm{MG}$, in which uncertainties of wind power production and environmental issues are remarked. As well, they suggested a probability-based idea to determine the probability, in which the MG is able to satisfy the demanded power in a self-sufficient way called as the probability of selfsufficiency (PSS). A mixed-integer linear programming method is suggested in [17] to obtain the optimum annual scheduling for an autonomous MG containing WTs, PV systems, ESSs, and diesel-based generation units. In [16], short-term optimum programming of an MG is discussed. They regarded various limitations of unit commitment for distributed generation such as ramp rate constraint and min-up and min-down times, as well as uncertain parameters. The authors in [18] employed the rolling horizon method for obtaining the optimum operation of a renewable MG. They mainly aimed to keep the balance among the supplement, load, and feeding a water pump for preserving the amount of water in a tank inside a predetermined range with the lowest expenditure. Furthermore, the authors in [19] utilized a TS (Tabu search) optimization method for obtaining the optimum operation for storage systems for a distribution system. They considered the uncertainty of EVs (electric vehicles). As well, the authors in [19] utilized the fuzzy sets idea for uncertainties modeling. The authors in [20] employed the fuzzy chance constraint programming (CCP) to program the production units of an MG in the dayahead platform. This MG contains distributed generations, WTs, loads, and uncertainties of loads. They presented the accessible wind energy in the form of the trapezoidal fuzzy set. An optimized fuzzy method is suggested in [21] for obtaining the optimum operation of a network containing WTs, PV systems, hydro, and heating-based generation units. This reference utilized fuzzy sets to consider the uncertainties of wind speed, irradiation, and accessible water. Then, the optimum answer is obtained by GA (genetic algorithm). Faddel et al. [22] also used an optimized fuzzy method to maximize the revenue of the considered parking lot operator, and fuzzy sets are utilized to consider the uncertain parameters like rates of the power market, state of batteries, and the departure time. Moreover, Rezvani et al. [23] aimed to schedule the power resources of an MG containing PV systems, FCs, ESSs, WTs, and microturbines. They used a multiobjective (MO) optimization method to find the best programming of the MG to reduce the entire operational expenditure and emitted pollutions down to the minimum possible amount, where the decision-making is conducted by the fuzzy method.

Based on the review papers, fuzzy methods, as well as stochastic ones, are beneficial to apply the uncertainty on the MGs. These two types of methods have a number of features and advantages. According to [24], for uncertainty with a normal distribution, stochastic methods will be useful, but in case of a general one, stochastic scheduling cannot mostly be 
solved simply. Besides, the fuzzy methods are useful even once the uncertainty is confined no regard to the unimodal distribution type. Generally, solution of the fuzzy scheduling problems is simpler compared to the stochastic ones. Also, Inuiguchi [25] represents that, for independent uncertain parameters, just a low count of decision-variables will be nonzero in the optimum answer of the fuzzy scheduling problem. However, in the case of the stochastic problems, many of them are nonzero in the optimum answer. With respect to the stated superiorities of the fuzzy idea, we utilized the fuzzy scheduling methods for solving the considered programming problem in this paper. The basic novelties of our suggested approach can be presented as follows:

(a) The considered uncertainties in wind/solar energy production and demanded power are modeled by fuzzy sets idea.

(b) The green power subsidies and balance penalties are considered here.

(c) This paper also regarded the uncertainties of the maximum charging/discharging capacity of the ESSs as well as the generated power of the FCs (that happens because of the oscillations in its temperature and exhausting) by fuzzy sets idea.

(d) The proposed fuzzy method can be improved by optimizing the free parameters using intelligent algorithms.

(e) Present work considered a bounded distribution line to exchange power with the power market. This line can assist the MG to sell the surplus produced electricity to the power market and decrease the probability of the load shedding through purchasing an amount of the required power from the power market in the lessen price times.

(f) Here, various uncertain parameters are implemented in the form of the fuzzy inequalities, and the fuzzy MILP method is suggested to schedule the MG. Also, an effective method with low complication is used here to optimize the fuzzy system.

Rest of the present work is structured in the following form: Section 2 models the proposed system. Then, various uncertain parameters are inserted in the model by fuzzy sets idea in Section 3. Section 4 provides the obtained results and analyzed them. Following that, Section 5 discusses around sensitivity factor for some variables with high importance. Eventually, Section 6 concludes the whole of the paper.

\section{Modeling of the System}

This paper considered an MG including WTs, PV systems, FCs, and ESSs. Figure 1 depicts the considered system of this paper. In this system, green power sources like WTs and PV systems are prior for supplying the loads. Also, this MG is considered in a grid-connected condition, and its connection is through a bounded distribution line. Once the generated power by these green power sources is not enough to satisfy the demanded power, inserted ESSs and FCs compensate the power shortage. Moreover, excess power of MG can be sold to the power market in the form of a virtual power plant (VPP). Expenditure of the received power from the main grid and the revenue of the sold power to it are specified with respect to the hourly tariffs of power market and also various plans of the main grid and MG owners. This work considered that the MG updates its manner in each hour. However, the demanded power, output power of green power sources, and tariffs of the power market are fixed during the periods.

$$
P_{p h_{t}} \leq P_{p h_{t}}^{\max }, \quad \forall t \in T .
$$

2.1. Green Power Sources. The wind power and solar radiation are two most broadly utilized green power sources of electricity generation. The generated electricity of the WTs is dependent on the wind speed and its power profile. As well, the generated electricity of PV systems is dependent on the installed capacity and the solar radiation. The output power of the green power sources cannot be exactly estimated, and always, an amount of error exists for a predicted value of their generated power, which is one of the most important challenges in the application of such resources. This prediction error in case of the PV systems is lower compared to the WTs because of the probability to utilize the historical information. Therefore, to achieve an effective and accurate model, uncertain factors of wind power and solar irradiation must be taken into account. This paper considered that the wind power and solar radiation are predicted for hours of the following day they are considered fixed during each period. The produced power of WTs and PV systems in a period $(t)$ must meet the following limitations:

$$
P_{w_{t}} \leq P_{w f_{t}}^{\max }, \quad \forall t \in T,
$$

Here, $P_{w_{t}}$ is the output electricity of WTs and $P_{w f_{t}}^{\max }$ denotes the maximum predicted output power of WTs. Equally, $P_{p h_{t}}$ and $P_{p h_{t}}^{\max }$ indicate the output and maximum predicted output of $\mathrm{PV}$ systems in period $t$. As well, $T$ signifies the set of the periods. This paper utilized the trapezoidal fuzzy sets to model the uncertainty of the predicted output powers that is presented in Section 4. Furthermore, some solar-based power production technologies exist such as solar-thermal ones, which can be remarked in the used model. Similarly, these solar-based plants use the solar power for electrical power generation, and so the uncertain factors of the radiation result in uncertainty of these plants. In equation (2), just the output of the solarbased power production plant is considered, and other technologies should be modeled in a similar manner.

Despite many advantages of green power sources, they have high installation expenditure. Various subsidies are planned by the governments for owners of such technologies as encouragements, in order to motivate the financiers to invest in this realm. Feed in tariff (FIT) is a famous one of these subsidies. The FIT is, in fact, a certain power price paid 


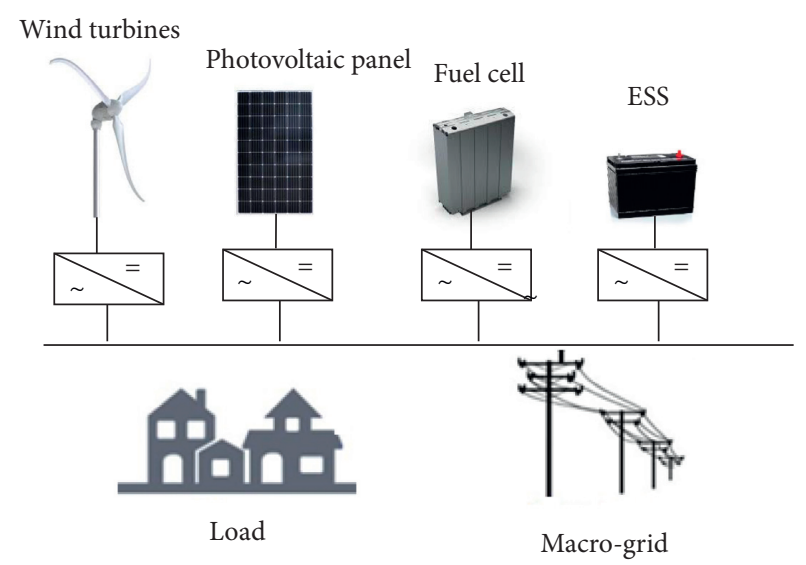

Figure 1: Proposed structure and components in the considered microgrid.

to the investors of green power sources for each generated power unit. This FIT in wind power and solar irradiation are regarded as a negative factor in the operational expenditure of WTs and PV systems.

This paper neglected the dynamic limitations of green power sources for facilitation.

2.2. ESS Systems. In renewable-based MGs, energy storage systems have high importance in compensation of prediction errors of green power sources and loads and reduction of load mismatches. If the real output power of these sources is higher than the prediction value, the storage systems will be charged, and they will store the extrapower. This saved electricity will be discharged in the energy shortage periods. Charge and discharge of the storage system is highly dependent on its capacity. So, the production constraints of the storage systems are presented as

$$
P_{E S S}^{\min } \leq P_{E_{S S}} \leq P_{E S S}^{\max }, \quad \forall t \in T,
$$

in which $P_{E S S}$ denotes the condition of storage system at the end of each period $(t)$. Also, $P_{E S S}^{\min }$ and $P_{E S S}^{\max }$ indicate, respectively, the min and max capacity of the storage system. The min capacity in this work is set to 0 . The max capacity is mostly a fixed value, but with growth of the battery life, the max capacity may reduce, and it cannot be precisely specified. Thus, the max capacity is presented in the form of a fuzzy set with a much smaller fuzziness limit in comparison with green power sources. Besides, the rate of charging/ discharging is bounded in the following form:

$$
\begin{gathered}
P_{E S S_{t}}^{\mathrm{dc}} \leq X_{t} * P_{E S S \text { max }}^{\mathrm{dc}}, \quad \forall t \in T, \\
P_{E S S_{t}}^{\mathrm{ch}} \leq Y_{t} * P_{E S S \text { max }}^{\mathrm{ch}}, \quad \forall t \in T, \\
X_{t}+Y_{t} \leq 1, \quad \forall t \in T .
\end{gathered}
$$

In these equations, $P_{E S S_{t}}^{\mathrm{dc}}$ and $P_{E S S_{t}}^{\mathrm{ch}}$ denote the discharged power and charged one, respectively; $P_{E S S \max }^{\mathrm{dc}}$ and $P_{E S S \text { max }}^{\mathrm{ch}}$ indicate the max rate of the ESS discharging and charging, respectively. As well, $X$ and $Y$ are regarded here avoiding from charging and discharging coincide. In addition, the max charging and discharging bounds of a storage system in period $t$ rely on its state storage in the prior period $(t-1)$ that is as follows [8]:

$$
\begin{gathered}
P_{E S S_{t}}^{\mathrm{ch}} * \delta^{\mathrm{ch}}+P_{E S S_{t-1}} \leq P_{E S S}^{\mathrm{max}}, \quad \forall t \in T, \\
\frac{P_{E S S_{t}}^{\mathrm{dc}}-P_{E S S_{t-1}} \leq 0, \quad \forall t \in T,}{\delta^{\mathrm{dc}}}, \quad,
\end{gathered}
$$

where $\delta^{\text {ch }}$ and $\delta^{\mathrm{dc}}$ denote the performances of ESS charging and discharging, respectively. Furthermore, the balance relation and initializing condition of a storage system can be presented as follows:

$$
P_{E S S_{t}}=P_{E S S_{t-1}}-\frac{P_{E S S_{t}}^{\mathrm{dc}}}{\delta^{d c}}+P_{E S S_{t}}^{\mathrm{ch}} * \delta^{\mathrm{ch}}, \quad \forall t \in T,
$$

in which $P_{I}$ denotes the initial saved electricity in the storage system.

2.3. FC System. An FC converts the chemical energy directly to the electrical power utilizing hydrogen and oxygen with low emission. The output power of the FCs must be less than its max power boundary that is defined by its manufacturer. This limitation can be presented as

$$
P_{f c_{t}} \leq P_{f c}^{\max }, \quad \forall t \in T
$$

In this formula, $P_{f_{c_{t}}}$ denotes the output power of the FC and $P_{f_{c}}^{\max }$ is the max capacity of the FC. Because the FC performance relies on some parameters like temperature and its lifetime, the max capacity of FC cannot be precisely indicated. Therefore, the upper bound of FC output power is defined in the form of a fuzzy number. This work considered that the power production of FCs is prior in comparison with the power production of the green power sources and ESSs.

2.4. Grid Connections. In this paper, a connection is considered between the MG and the upper grid through a distribution line with a bounded capacity. As stated, the extra power of the MG can be sold to the power market, and its required power can be provided from the power market. In addition, the MG receives/gives the market prices for sold/purchased power to/from the power market. The limitations of this connection line are presented as

$$
\begin{aligned}
& P_{\text {sell }_{t}} \leq P_{L}^{\max }, \quad \forall t \in T, \\
& P_{\text {buy }_{t}} \leq P_{L}^{\max }, \quad \forall t \in T,
\end{aligned}
$$

in which $P_{\text {buy }_{t}}$ and $P_{\text {sell }}$ are, respectively, the purchased and sold power from and to power market in $t$ period. Also, $P_{L}^{\max }$ denotes the max heat capacity of this line. The power tariffs are considered as constant known values in each period. Here, the MG can perform its own scheduling, in a way that receives the max profit from power market alongside minimization of its entire power production expenditure. 
2.5. Loads. An active load is considered here that is not elastic. The mean hourly load also has a constant value. The uncertainty is not involved in the basic scenario of the load, while it is furthered and analyzed in the next sections. The implemented distributed generations probably cannot satisfy all demanded power. In such conditions, more prior loads will be fed. Balance relations of produced power with used power for each period can be given as

$$
P_{w_{t}}+P_{p h_{t}}+P_{E S S_{t}}^{\mathrm{dc}}-P_{E S S_{t}}^{\mathrm{ch}}+P_{f c_{t}}-P_{\text {sell }_{t}}+P_{\text {buy }_{t}}+P_{u_{t}}=P_{\text {load }_{t}},
$$

$\forall t \in T$,

where $P_{u_{t}}$ denotes the curtailed electricity of the MG and $P_{\text {load }_{t}}$ indicates the used electricity at period $t$.

\section{Suggested Model}

The optimum programming problem of the $\mathrm{MG}$ in the absence of the uncertain parameters can be presented in the form of a MILP model:

$$
\begin{aligned}
\min f_{0}= & \sum_{t=1}^{24} \Delta t *\left(P_{w_{t}} * c_{w}+P_{p h_{t}} * c_{p h}+P_{E S S_{t}}^{\mathrm{dc}} * c_{d c}\right. \\
& -P_{E S S_{t}}^{\mathrm{ch}} * c_{\mathrm{ch}}+P_{f c_{t}} * c_{f c}+P_{\mathrm{buy}_{t}} * c_{\text {buy }_{t}} \\
& \left.-P_{\text {sell }_{t}} * c_{\text {sell }_{t}}+P_{u_{t}} * c_{u}\right),
\end{aligned}
$$

s.t:

in equations (1)-(14).

In these equations, $\Delta t$ denotes the period's length ( 1 hour is considered here); $c_{w}, c_{p h}$, and $c_{f c}$ indicate the production expenses of the WTs and PV systems and FCs; and $c_{\mathrm{dc}}$ and $c_{\mathrm{ch}}$ are the expense and profit allocated with the battery discharging and charging, respectively. Also, $c_{\text {buy }_{t}}$ and $c_{\text {cell }_{t}}$ are, respectively, the tariffs for purchased and sold power from/to the power market at period $t$. So, for wind power and sun irradiation, factors $c_{w}$ and $c_{p h}$ in relation (15) can be substituted with

$$
\begin{aligned}
c_{w}^{\prime} & =c_{w}-\lambda_{w}, \\
c_{p h}^{\prime} & =c_{p h}-\lambda_{p h},
\end{aligned}
$$

where $\lambda_{w}$ and $\lambda_{p h}$ are, respectively, the constant subsidies, which the MG receives per generated power by WTs and PV systems.

As stated earlier, an MG contains various uncertain parameters that must be remarked in the modeling process. Let a crisp mixed-integer linear program problem be expressed in the following form:

$$
\begin{aligned}
& \min c^{T} x, \\
& \text { s.t. } \\
& A_{\text {eq }} x=b_{\text {eq }}, \\
& A_{\text {ineq }} x \leq b_{\text {ineq }}, \\
& \quad x \geq 0 .
\end{aligned}
$$

In case of the existence of fuzzy numbers in the vectors/ matrices $\left(\left\{c, b_{\text {eq }}, b_{\text {ineq }}\right\} /\left\{A_{\text {eq }}, A_{\text {ineq }}\right\}\right)$, the problem will be a fuzzy mixed-integer linear programming. Various approaches are suggested for this kind of problems (fuzzy MILP). With respect to Section 2, the uncertainties of the green power sources generated electricity, battery max capacity, FC output electricity, and the max capacity of the distribution network occur in the max allowable values of equations (1)-(5) and equations (7) -(9), respectively. So, the fuzzy MILP problem is expressed as

$$
\min c^{T} x
$$

s.t.

$$
\begin{gathered}
A_{\text {eq }} x=b_{\text {eq }}, \\
A_{\text {ineq }} x \leq \bar{b}_{\text {ineq }}, \\
x \geq 0,
\end{gathered}
$$

in which $\bar{b}_{\text {ineq }}$ is the fuzzy section of the problem. Differences of the crisp inequality with fuzzy one is captured in Figure 2 for an instance. In Figure 2(a), the max allowable value (denoted by $b$ ) denotes a crisp factor that results in a crisp inequality. In Figure 2(b), the exact value of $b$ parameter cannot be specified. So, the feasible set denotes a fuzzy set. As can be seen from Figure 2(c), we can devote a trapezoidal MF (membership function) for mentioned fuzzy set. All trapezoidal fuzzy sets (similar to Figure 2(c)) are dividable into two regions including a crisp one (where $\mu(x)=1$ ) and a fuzzy region (where $\mu(x)$ is lower than one), which are shown in Figure 2(c) using $b_{0}$ and $r$ factors. For instance, in case of exact prediction of WT output electricity, the max limitation of WT output electricity will be $P_{w f_{t}}^{\max }$, and if the exact output power cannot be predicted, this limitation will be $\bar{P}_{w f}^{\max }$ that can be separated into two crisp and fuzzy regions (denoted by $P_{w f_{t} 0}^{\max }$ and $r_{w}$, respectively). The lower bound also will be similar.

Many methods are presented in literature for fuzzybased linear optimization problems solving [4-7]. According to the construction of the suggested model in equations (18)-(21), where just $\bar{b}_{\text {ineq }}$ has fuzzy values, the suggested approach in [7] is employed here for solving the considered fuzzy problem. The fuzzy-based optimization problems in [4-6] are solved by a higher number of fuzzy variables like fuzzy values of vectors $c$ and $b_{\mathrm{eq}}$ and matrices $A_{\text {eq }}$ and $A_{\text {ineq. }}$. In this paper, the mentioned parameters have been optimized by an intelligent algorithm which is introduced in the next section. This higher number of fuzzy parameters results in very complicated approaches that are not essential to solve the presented model in equations (18)-(21).

The base of the suggested method in [7] is the generalized form of Bellman and Zadeh (1970), in which a fuzzy decision $(D)$ is introduced in a fuzzy set form generated by intersection of the fuzzy limitations $C_{i}, i=1,2, \ldots, m$. Here, $m$ denotes the count of the limitations and the fuzzy goal $(G)$. Assuming that these limitations and $G$ are categorized using the trapezoidal MFs $\left(\mu_{C_{i}}(x), i=1, \ldots, m\right.$, the fuzzy decision will also be categorized using the MF as follows: 


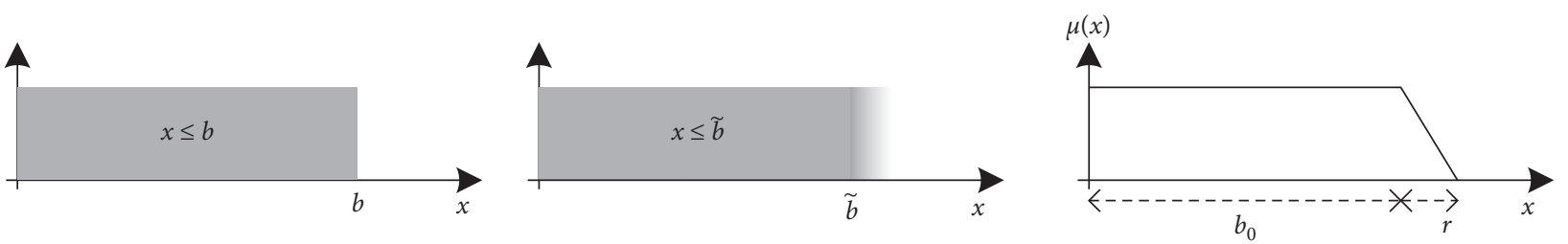

FIGURE 2: General presentation of (a) crisp inequality, (b) fuzzy inequality, and (c) membership functions of the fuzzy inequality.

$$
\mu_{D}(x)=\min \left\{\mu_{C_{1}}(x), \ldots, \mu_{C_{m}}(x), \mu_{G}(x)\right\} .
$$

Here, an optimum decision is presented as a solution $x$ that can minimize the $\mu_{D}(x)$. Primarily, the trapezoidal MFs must be known for obtaining the optimum decision. Also the memberships of limitations are structured with respect to the stated descriptions and Figure 2. For obtaining the MF of the $G$, two following optimal problems should be solved to obtain the extreme points of the cost function:

$$
\begin{aligned}
& f^{0}=\min _{x \in X_{1}} c^{T} x, \\
& f^{1}=\min _{x \in X_{2}} c^{T} x,
\end{aligned}
$$

in which

$$
\begin{aligned}
& X_{1}=\left\{x \in R^{n} \mid A_{\mathrm{eq}} x=b_{\mathrm{eq}} \cdot A_{\text {ineq }} x \leq b_{\text {ineq } 0}, x \geq 0\right\}, \\
& X_{2}=\left\{x \in R^{n} \mid A_{\text {eq }} x=b_{\text {eq }} \cdot A_{\text {ineq }} x \leq b_{\text {ineq } 0}+r, x \geq 0\right\} .
\end{aligned}
$$

Also, $f^{0}$ and $f^{1}$ denote, respectively, the max and min points for the cost function. Then, the optimum solution minimizing the $\mu_{D}(x)$ is obtained using the following formulations:

$$
\min \theta
$$

s.t.

$$
\begin{aligned}
A_{\mathrm{eq}} x & =b_{\text {eq }}, \\
c^{T} x & \leq f^{1}-\left(f^{1}-f^{0}\right) \theta, \\
A_{\text {ineq }} x & \leq b_{\text {ineq } 0}+r \theta, \\
x & \geq 0, \quad \theta \in[0,1] .
\end{aligned}
$$

Here, $\theta$ denotes a secondary parameter that is comprehensively described in [7].

So, the optimum programming is obtained by the stated process. Because just the crisp section of all inequality limitations is regarded in the first optimization, the following formulation is presented:

$$
\begin{aligned}
\min f^{0}= & \sum_{t=1}^{24} \Delta t *\left(P_{w_{t}} * c_{w}^{\prime}+P_{p h_{t}} * c_{p h}^{\prime}+P_{E S S_{t}}^{\mathrm{dc}} * c_{d c}\right. \\
& -P_{E S S_{t}}^{\mathrm{ch}} * c_{c h}+P_{f_{t}} * c_{f c}+P_{\text {buy }_{t}} * c_{\text {buy }_{t}} \\
& \left.-P_{\text {sell }_{t}} * c_{\text {sell }_{t}}+P_{u_{t}} * c_{u}\right)
\end{aligned}
$$

s.t.

$$
\begin{aligned}
& P_{w_{t}} \leq P_{w f_{t} 0}^{\max }, \quad \forall t \in T, \\
& P_{p h_{t}} \leq P_{p h_{t} 0}^{\max }, \quad \forall t \in T, \\
& P_{E S S_{t}} \leq P_{E S S 0}^{\max }, \quad \forall t \in T, \\
& P_{E S S_{t}}^{\mathrm{ch}}+P_{E S S_{t-1}} \leq P_{E S S 0}^{\max }, \quad \forall t \in T, \\
& P_{f_{c_{t}}} \leq P_{f c 0}^{\max }, \quad \forall t \in T \\
& P_{\text {sell }_{t}} \leq P_{L 0}^{\max }, \quad \forall t \in T, \\
& P_{\text {buy }_{t}} \leq P_{L 0}^{\max }, \quad \forall t \in T .
\end{aligned}
$$

According to equations (4)-(6), (8)-(10), and (14), we can write

$$
\forall t \in T \text {, }
$$

in which $T$ denotes the set of the intervals. The max probable amount for each one of the inequality limitations in the $2^{\text {nd }}$ optimization is regarded, and the optimization is presented by

$$
\begin{aligned}
\min f^{1}= & \sum_{t=1}^{24} \Delta t *\left(P_{w_{t}} * c_{w}^{\prime}+P_{p h_{t}} * c_{p h}^{\prime}+P_{E S S_{t}}^{\mathrm{dc}} * c_{\mathrm{dc}}\right. \\
& -P_{E S S_{t}}^{\mathrm{ch}} * c_{\mathrm{ch}}+P_{f c_{t}} * c_{f c}+P_{\text {buy }_{t}} * c_{\text {buy }_{t}} \\
& \left.-P_{\text {sell }_{t}} * c_{\text {sell }_{t}}+P_{u_{t}} * c_{u}\right)
\end{aligned}
$$

s.t.

$$
\begin{aligned}
& P_{w_{t}} \leq P_{w f_{t} 0}^{\max }+r_{w}, \quad \forall t \in T, \\
& P_{p h_{t}} \leq P_{p h_{t} 0}^{\max }+r_{p h}, \quad \forall t \in T, \\
& P_{E S S_{t}} \leq P_{E S S 0}^{\max }+r_{E S S}, \quad \forall t \in T, \\
& P_{E S S_{t}}^{\mathrm{ch}}+P_{E S S_{t-1}} \leq P_{E S S 0}^{\max }+r_{E S S}, \quad \forall t \in T, \\
& P_{f c_{t}} \leq P_{f c 0}^{\max }+r_{f c}, \quad \forall t \in T, \\
& P_{\text {sell }_{t}} \leq P_{L 0}^{\max }+r_{L}, \quad \forall t \in T, \\
& P_{\text {buy }_{t}} \leq P_{L 0}^{\max }+r_{L}, \quad \forall t \in T .
\end{aligned}
$$

According to equations (4)-(6), (8)-(10)-(14), we can write 
$\forall t \in T$.

Eventually, the best solution is obtained using the following formulation:

$\min \theta$,

$$
\begin{aligned}
& \text { s.t. } \\
& \sum_{t=1}^{24} \Delta t *\left(P_{w_{t}} * c_{w}^{\prime}+P_{p h_{t}} * c_{p h}{ }^{\prime}+P_{E S S_{t}}^{\mathrm{dc}} * c_{\mathrm{dc}}-P_{E S S_{t}}^{\mathrm{ch}} * c_{\mathrm{ch}}\right. \\
& +P_{f c_{t}} * c_{f c}+P_{\text {buy }_{t}} * c_{\text {buy }_{t}}-P_{\text {sell }_{t}} * c_{\text {sell }_{t}}+P_{u_{t}} * c_{u} \\
& \left.+P_{e_{t}} * c_{e}\right) \leq f^{1}-\theta *\left(f^{1}-f^{0}\right), \\
& P_{w_{t}} \leq P_{w f_{t} 0}^{\max }+\theta r_{w_{t}}, \quad \forall t \in T \\
& P_{p h_{t}} \leq P_{p h_{t} 0}^{\max }+\theta r_{p h_{t}}, \quad \forall t \in T, \\
& P_{E S S_{t}} \leq P_{E S S 0}^{\max }+\theta r_{E S S}, \quad \forall t \in T, \\
& P_{E S S_{t}}^{\mathrm{ch}}+P_{E S S_{t-1}} \leq P_{E S S 0}^{\mathrm{max}}+\theta r_{E S S}, \quad \forall t \in T, \\
& P_{f_{c}} \leq P_{f c 0}^{\max }+\theta r_{f c}, \quad \forall t \in T \\
& P_{\text {sell }_{t}} \leq P_{L 0}^{\max }+\theta r_{L}, \quad \forall t \in T, \\
& P_{\text {buy }_{t}} \leq P_{L 0}^{\max }+\theta r_{L}, \quad \forall t \in T .
\end{aligned}
$$

According to equations (4)-(6), (8)-(10), and (14), we can write

$$
\forall t \in T \text {. }
$$

3.1. Proposed Optimization Algorithm. The main purpose of the optimization problem is similar to the whales' collective movement to achieve a common goal. Hence, modeling their movement as a new algorithm is a suitable method for optimization problems [26]. The algorithm studied in this paper, which modes based on the whale's behavior, is called sperm whales swarm optimization (SWSO) algorithm. Each group of whales consists of 50 members, and one of the whales is chosen as a leader of the group, and another member should follow him. The leaders of all groups are also in contact together, which is shown in Figure 3. Based on the closest whale to the target, the leader of the group is selected. At the time of the grouping movement of the whales, there is the possibility of changing the leader of the subgroup and the main group. follows:

The steps to implement the SWSO algorithm are as

Step 1. A number of sperm whales are randomly created in each group (SW), and $S W$ is in the range of $1 \leq s w \leq 10$.

Step 2. The fitness is selected according to the position of each sperm for SW of the population.
Step 3. The current position of each particle is compared with the best previous position of all particles and their necessary replacement in $n$ particles, and the best position of the particle is selected:

$$
\text { If } F\left(P_{i}^{n}\right)>\text { pbest }_{i}^{n} \Longrightarrow\left\{\begin{array}{l}
\text { pbest }_{i}^{n}=F\left(P_{i}^{n}\right), \\
\vec{x} \text { pbest } 0_{i}^{n}=\vec{x}_{i}^{t}(t) .
\end{array}\right.
$$

Step 4. The current position of each particle is compared with the best previous position of all particles and location in $n$ particles, and the best position of the particle is selected:

$$
\text { If } F\left(P_{i}^{n}\right)>\text { gbest }^{n} \Longrightarrow\left\{\begin{array}{l}
\text { gbest }^{n}=F\left(P_{i}^{n}\right) \\
\vec{x} \text { gest }^{n}=\vec{x}_{i}^{t}(t)
\end{array}\right.
$$

Step 5. The current position of each particle is compared with the best previous position of all particles and necessary location in $n$ particles, and the best position of the particle is selected:

$$
\text { If } F\left(P_{i}^{n}\right)>h \text { best } \Longrightarrow\left\{\begin{array}{l}
h \text { best }=F\left(P_{i}^{n}\right), \\
\vec{x} \text { gbest }=\vec{x}_{i}^{t}(t)
\end{array}\right.
$$

Step 6. For each of the particles in $n$ population, the velocity vector is computed using the following equation:

$$
\begin{aligned}
v_{i d}(t+1)= & w \cdot v_{i d}(t)+c_{1} \cdot \operatorname{rand}\left(p_{-} \text {best }_{\mathrm{id}}-x_{i d}\right) \\
& +c_{2} \cdot \operatorname{rand}\left(h_{-} \text {best }_{d}-x_{i d}\right)
\end{aligned}
$$

where h_best is the best answer obtained from the comparison among the g_best and selected the smallest of them.

Step 7. The new position of each particle in $n$ population is calculated from the following equation:

$$
x_{i d}(t+1)=x_{i d}(t)+v_{i d}(t+1)
$$

Step 8. If convergence is reached, it stops; otherwise, it returns to step 2 .

In equation (58), inertia weight is represented by $w$. Also, $c_{1}$ is and $c_{2}$ are training factors, respectively, and are equal to 2 . The degree of convergence is proportional to $w$ and is defined based on it. In the evolution of the population process, the linear trend is down from 0.4 to 0.9 . Initially, the higher values are more appropriate to achieve best answers. On the other hand, small values $w$ increase the convergence rate and obtain 


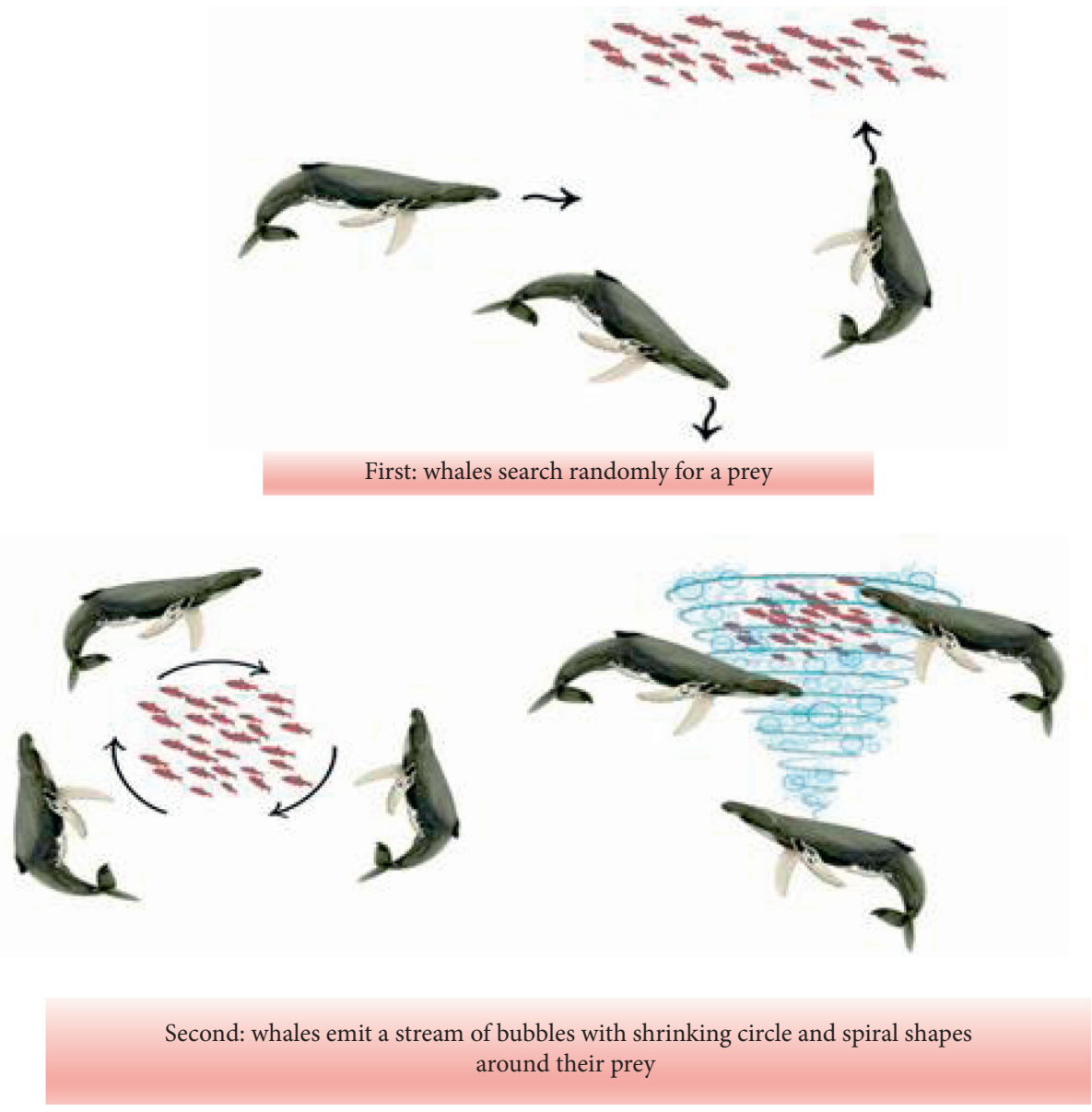

FIgURE 3: A group of three whales.

better results for convergence. This downward trend is defined using the following equation:

$$
w=w_{\max }-\frac{w_{\max }-w_{\min }}{\text { iter }_{\max }} \times \text { iter. }
$$

Two groups $(n=2)$ are assumed in the purposed algorithm for this study.

By using various mathematical functions, examining the performance of the proposed algorithm can be analyzed. In this paper, the jinc function is proposed to evaluate the performance of the proposed algorithm. The jinc function has a kind of sample Fourier transform. For large values of $X$, jinc $(t)$ is done a damped, shifted cosine. Specifically, it is defined as follows:

$$
\operatorname{jinc}(x) \approx \cos \left(|x|-\frac{3 \pi}{4}\right) \sqrt{\frac{2}{\pi|t|^{3}}} .
$$

The three-dimensional structure of the jinc function is depicted in Figure 4. As seen, this function has many local points and also has only one global point. This function is embedded in the proposed algorithm, and then, the global maximum point is obtained. In Table 1, the results of the implementation of 10 independent runs in the program are listed using the proposed algorithm and the optimization of the jinc function with the assumption $f(x)=1$.

By examining the results given in Table 1 , it can be stated that the best answer given by the jinc function is 0.99998 . The mean value of best $F(x, y)$ in 10 iterations is 0.99976 , where it achieved in average 0.1393 seconds. 0.99875 is the highest deviation. The obtained values for each iteration are plotted in Figure 1. The convergence of two independent groups of SWSO algorithms is depicted in Figure 5.

To counteract the problem of overcongestion of lines (density management), the SWSO algorithm is proposed.

\section{Test System}

This part of the paper implemented the suggested approach on a practical test system [8]. Firstly, the case study is optimally scheduled by the attainable information. Following that, effects of the various factors of the MG over the case study are computed and also analyzed.

4.1. Case Study Presentation. An MG is considered here as the case study located in Budapest Tech that is represented earlier in [8]. The considered microgrid contains different elements, including WTs, PV systems, a fuel cell, a battery, and loads. The max generable power by WTs and PV 


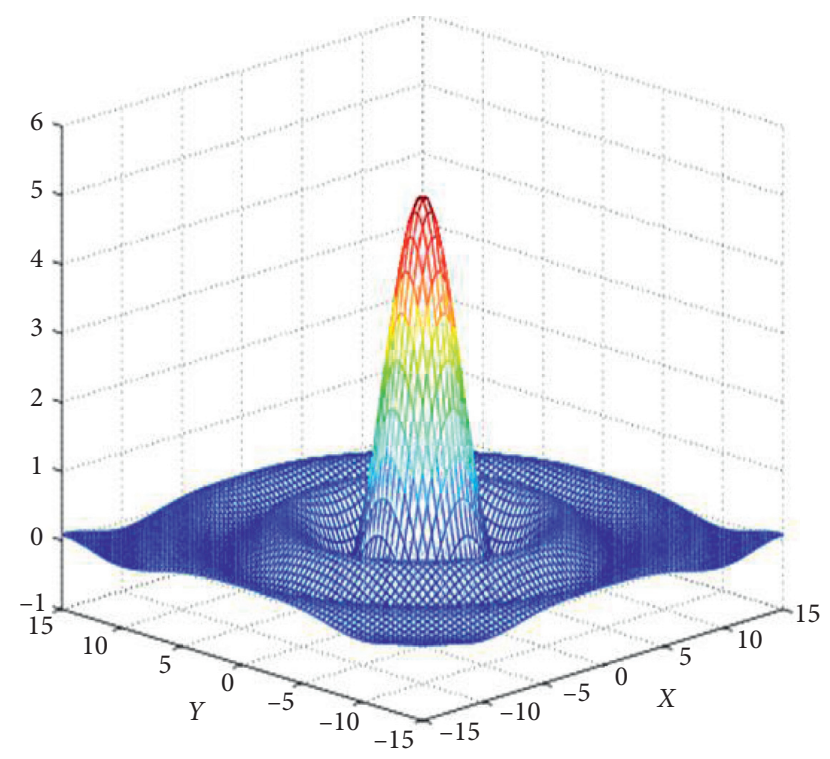

FIgURE 4: The three-dimensional jinc function.

TABle 1: Results of 10 independent run by SWSO algorithm.

\begin{tabular}{lcc}
\hline Implementation & SWSO algorithm & Error \\
\hline 1 & 0.99975 & 0.00025 \\
2 & 0.99998 & 0.00002 \\
3 & 0.99980 & 0.0002 \\
4 & 0.99875 & 0.00125 \\
5 & 0.99998 & 0.00002 \\
6 & 0.99995 & 0.00005 \\
7 & 0.99990 & 0.00010 \\
8 & 0.99988 & 0.00012 \\
9 & 0.99994 & 0.00006 \\
10 & 0.99995 & 0.00005 \\
Average & 0.99976 & 0.00195 \\
\hline Average time & \multicolumn{3}{c}{0.1393} \\
Best results & \multicolumn{2}{c}{0.99998} \\
\hline
\end{tabular}

systems is, respectively, $400 \mathrm{~kW}$ and $150 \mathrm{~kW}$. Also, the battery and FC capacities are considered $200 \mathrm{kWh}$ and 80 $\mathrm{kWh}$. Discharging and charging rates of the battery are $50 \mathrm{~kW} / \mathrm{h}$ and $200 \mathrm{~kW} / \mathrm{h}$, respectively. Table 2 listed the wind power, sun irradiation, mean hourly power market tariffs, and the demanded power for a certain day [8]. The considered MG is linked to the main grid via a distribution network with $30 \mathrm{~kW}$ max capacity. Some other parameters are regarded as

$$
r_{E S S}=5 \mathrm{~kW}, r_{f c}=3 \mathrm{~kW} \text { and } r_{L}=3 \mathrm{~kW} .
$$

Moreover, Table 3 provided the values of various expenditure factors. In this table, it is considered:

$$
\begin{aligned}
& c_{w}=c_{p h}=0.4 \$ / \mathrm{kWh}, \\
& \lambda_{w}=\lambda_{p h}=0.2 \$ / \mathrm{kWh} .
\end{aligned}
$$

Both charge and discharge performances of the battery are 100 percent.

4.2. Obtained Results for the Basic Scenario. Various considerations are regarded in obtaining the numerical results.

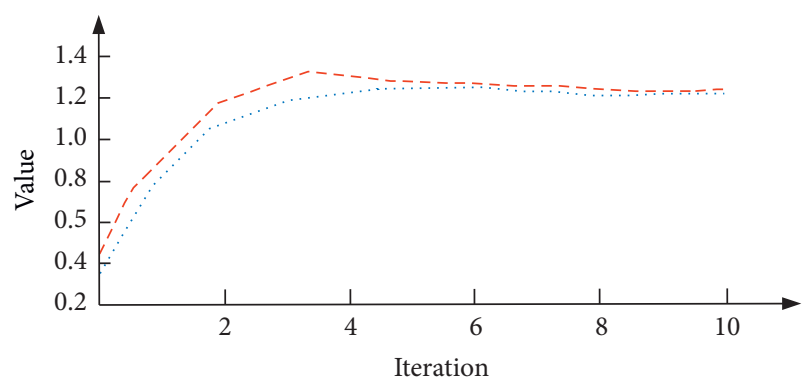

..... Iteration 3 , Table 1
- - - Iteration 6, Table 1

FIGURE 5: Convergence of two independent groups of SWSO algorithms.

The results for a scenario by considering the stated assumptions are obtained, which is depicted in Figures 6 and 7. In these results, the sold and purchased power tariffs are the same. This scenario is considered as the basic scenario. The entire expenditure of this scenario is obtained as $1317 \$$, and $\theta$ is 0.487 . According to the obtained results, the power tariffs in the first nine time interval are lower compared to the remaining intervals. So, one would like to purchase the power from the power market in the first nine hours and grow its profit through power selling in the following intervals. The power price in latest four-time interval of a day is great. On the contrary, the generated electricity of the PV systems is equal to 0 in this period, and the power usage is relatively high. Thus, the MG should satisfy the demanded power by purchasing the power from the power market. Because the FC unit is a highcost resource in comparison with the other resources of the MG, only this unit produces the electricity in the latest nine time interval of the day, once the power has a high price and the generated electricity by the green power sources reduces.

As can be seen from Figure 6, the MG cannot serve the total demanded power in hour 21, when the max generation of the MG is $279.8 \mathrm{~kW}$, while the total demanded power is $290 \mathrm{~kW}$.

4.3. Sensitivity Analysis for Various Factors. This part of the paper represented the sensitivity analyzing for various parameters of the MG to investigate the effects of various factors' variations over the optimum programming. The considered factors are presented as follows:

(a) The fuzziness parameter ( $r$ )

(b) Procedure of the MG operator for the green power subsidy

(c) Capacity of the batteries and FCs

(d) The max capacity of the connection line between the MG and the main grid

Table 4 represents the obtained results of the sensitivity analysis. Also, these results are analyzed in the following parts.

4.4. Effects of the Fuzziness Parameters Growth. The fuzziness variable can be used to model the growth of uncertainties. The MF variation for growth of the fuzziness parameter from $r_{1}$ to $r_{2}$ is depicted in Figure 8. The optimum scheduling of the MG can be influenced by the uncertainty level. For 
TABLE 2: Used data for the supplied power by green power sources, demands, and power market tariffs (hourly data).

\begin{tabular}{|c|c|c|c|c|c|c|}
\hline \multirow{2}{*}{ Time interval } & \multicolumn{2}{|c|}{ Wind power in $\mathrm{kW}$} & \multicolumn{2}{|c|}{ Solar power in $\mathrm{kW}$} & \multirow{2}{*}{ Power market tariff in $\$$ per $\mathrm{kWh}$} & \multirow{2}{*}{$P_{\text {load }_{t}}$ in $\mathrm{kW}$} \\
\hline & $P_{w f_{t} 0}^{\max }$ & $r_{w_{t}}$ & $P_{p h f_{t} 0}^{\max }$ & $r_{p h_{t}}$ & & \\
\hline 1 & 125.1 & 10.6 & 0.0 & 0.0 & 0.30 & 190 \\
\hline 2 & 143.8 & 11.9 & 0.0 & 0.0 & 0.20 & 170 \\
\hline 3 & 134.7 & 11.6 & 0.0 & 0.0 & 0.31 & 140 \\
\hline 4 & 154.2 & 13.1 & 0.0 & 0.0 & 0.38 & 110 \\
\hline 5 & 95.8 & 7.9 & 0.0 & 0.0 & 0.41 & 100 \\
\hline 6 & 114.9 & 9.4 & 4.7 & 0.2 & 0.47 & 140 \\
\hline 7 & 143.8 & 11.7 & 9.6 & 0.7 & 0.46 & 190 \\
\hline 8 & 173.0 & 14.2 & 28.8 & 2.0 & 0.46 & 210 \\
\hline 9 & 162.8 & 13.5 & 57.9 & 3.3 & 0.46 & 230 \\
\hline 10 & 153.3 & 13.1 & 96.7 & 5.8 & 0.48 & 250 \\
\hline 11 & 114.6 & 9.3 & 125.7 & 8.1 & 0.49 & 250 \\
\hline 12 & 125.0 & 10.7 & 136.2 & 8.6 & 0.50 & 260 \\
\hline 13 & 143.8 & 11.8 & 145.8 & 8.7 & 0.52 & 260 \\
\hline 14 & 169.1 & 13.9 & 136.2 & 8.2 & 0.53 & 240 \\
\hline 15 & 177.4 & 15.2 & 125.9 & 7.6 & 0.54 & 240 \\
\hline 16 & 115.5 & 9.3 & 96.8 & 5.6 & 0.56 & 250 \\
\hline 17 & 125.0 & 10.2 & 58.0 & 3.4 & 0.54 & 260 \\
\hline 18 & 134.1 & 10.9 & 28.9 & 2.0 & 0.58 & 280 \\
\hline 19 & 162.9 & 13.4 & 9.9 & 0.7 & 0.62 & 290 \\
\hline 20 & 182.7 & 15.6 & 5.2 & 0.2 & 0.64 & 300 \\
\hline 21 & 114.7 & 9.3 & 0.0 & 0.0 & 0.58 & 300 \\
\hline 22 & 162.8 & 13.5 & 0.0 & 0.0 & 0.60 & 280 \\
\hline 23 & 125.0 & 10.2 & 0.0 & 0.0 & 0.52 & 250 \\
\hline 24 & 143.8 & 11.8 & 0.0 & 0.0 & 0.47 & 240 \\
\hline
\end{tabular}

TABLE 3: Cost coefficients for various components of the microgrid.

\begin{tabular}{lccccc}
\hline$c_{w}^{\prime}$ in $\$ / \mathrm{kWh}$ & $c_{p h}^{\prime}$ in $\$ / \mathrm{kWh}$ & $c_{f c}$ in $\mathrm{kWh}$ & $c_{d c}$ in $\$ / \mathrm{kWh}$ & $c_{c h}$ in $\$ / \mathrm{kWh}$ & $c_{u}$ in $\$ / \mathrm{kWh}$ \\
\hline 0.2 & 0.2 & 0.5 & 0.3 & 0.2 & 1.5 \\
\hline
\end{tabular}

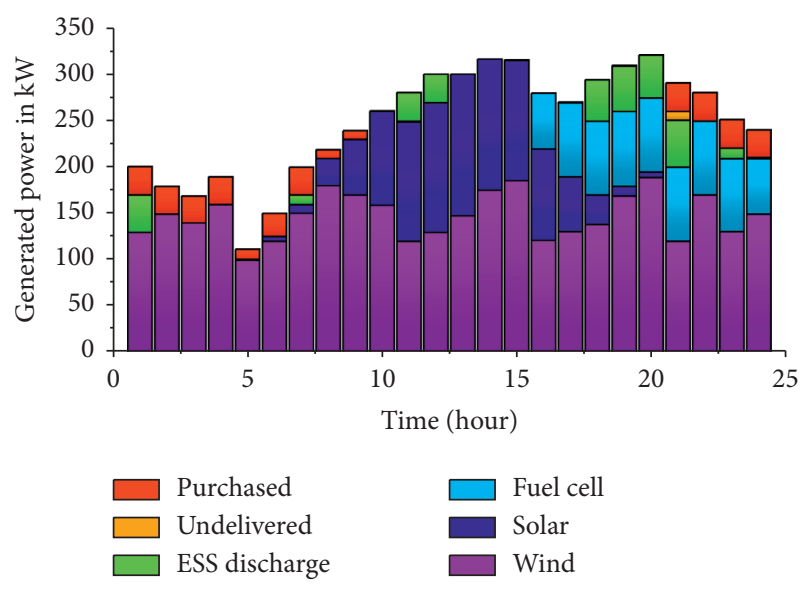

FIGURE 6: The optimum scheduling for the produced power of various power sources and purchased power from the power market (the basic scenario).

investigation of the uncertainty growth effects, it is considered that all of the fuzziness variables $\left(r_{w_{t}}, r_{p h_{t}}, \ldots\right)$ are multiplied by $\mathrm{M}$. Here, $M$ has a real positive value. Besides, according to Figure 8 , the crisp regions $\left(P_{w f_{t} 0}^{\max }, P_{p h_{t} 0}^{\max }, \ldots\right)$ must be substituted with $P_{w f_{t} 0}^{\max }+0.5 r_{w_{t}}(1-M)$,

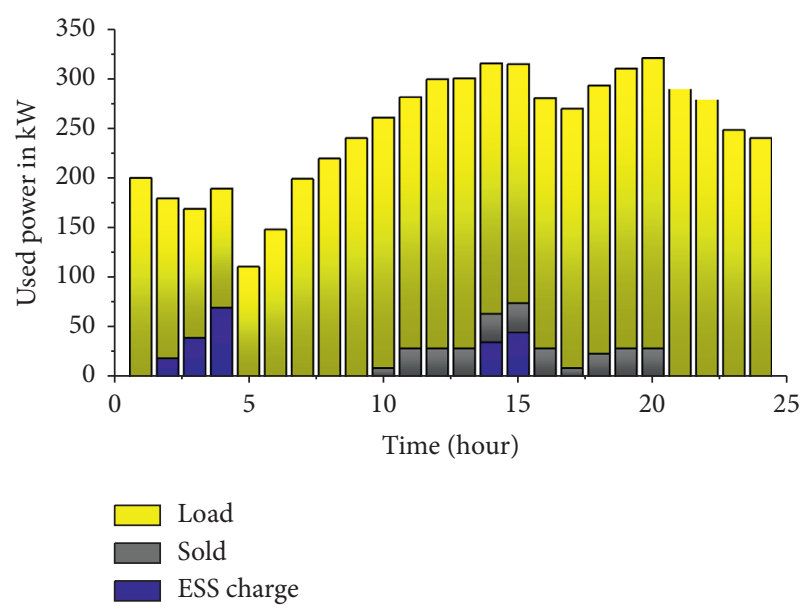

FIGURE 7: The optimum power consumption in the MG (the basic scenario).

$P_{p h_{t} 0}^{\max }+0.5 r_{p h_{t}}(1-M), \ldots$. Table 4 provides the obtained results of 3 various amounts of $M$ as cases 1 to 3 . Based on the results of these three cases, it can be claimed that growth of the $M$ in the range of $[0.1,10]$ (growth of the uncertainty) results in the following: 


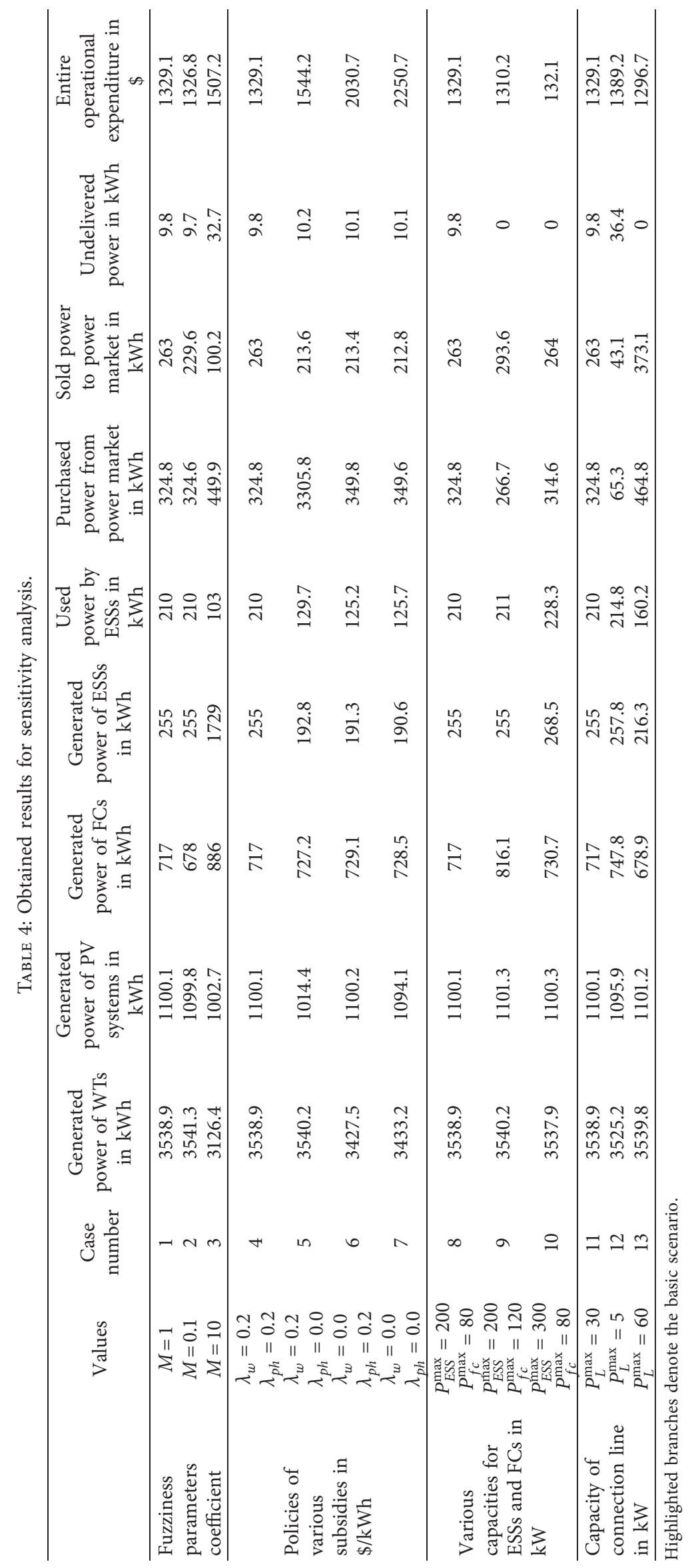


(1) The generated powers by WTs and PV systems decrease, respectively, down to 11.5 and 8.6 percent. In other words, by growth of the uncertainties, reliability of the green power sources will be decreased, and the MG will be forced to procure its power by resources with higher costs that inflict further expenses to the MG.

(2) The generated power of the FC grows up to 33 percent that results in growth of the operational expenditure.

(3) Power purchasing from the main grid raises around 38.5 percent, and power selling to the grid decreases down to 44.2 percent. In other words, the MG will be more dependent on the main grid. Besides, difference of the purchased and sold power increases around 575 percent, resulting in a lower profit for the MG.

(4) All stated events cause 13.5 percent growth in the entire operational expenditure of the MG.

Effects of the fuzziness parameters growth over the operational expenditure of the MG are depicted in Figure 9. In each stage of this process, just one of the fuzzy variables is multiplied by $M$. In this figure, the purple curve serves a scenario, where every fuzzy variable is multiplied by $M$ parameter. Based on this figure, the fuzzy parameter of the WT output has the highest importance over growth of the operational expenditure through fuzzy parameter growth. So, improvement of the wind speed prediction equipment is the best way to decrease the operational expenditure, here. This figure as well demonstrates that the operational expenditure has almost constant value once $M$ is lower than 2 . Thus, this range is not desirable for MG owner. Remarkably, when only one fuzzy parameter increases, the total expense variation has lower values, while the variation of total expenses is higher when all fuzzy parameters increase.

4.5. Effects of Variations in Supporting Policies of the MG. Supporting trends of MGs for green power sources are different for various networks. Present work considered that there is a constant subsidy (per $\mathrm{kWh}$ ) for power production of green sources. As stated, they are inserted in the formulations in relations (28), (37), and (47) through substitution of the factors $c_{w}, c_{p h}$ with $c_{w}^{\prime} c_{p h}^{\prime}$

Table 3 provides the effects of various policies over the MG programming for cases 4 to 7 . According to the results of cases 4-6, elimination of wind and solar power subsidies results in a reduction around $85.5 \mathrm{kWh}$ and $133.4 \mathrm{kWh}$, respectively, in scheduled power of PV systems and WTs. Also, case 4 depicts that $63 \%$ of the programmed power is procured by WTs, whereas around $19 \%$ of the daily demanded power is used by the PV systems. So, the effects of wind power subsidy elimination over the operational expenditure of the MG are higher than the solar energy subsidy elimination. As well, removing wind power subsidy has high importance over the growth of the operational expenditure of the MG to 53 percent in case 6 . In addition to the mentioned reason, growth of the operational expenditure

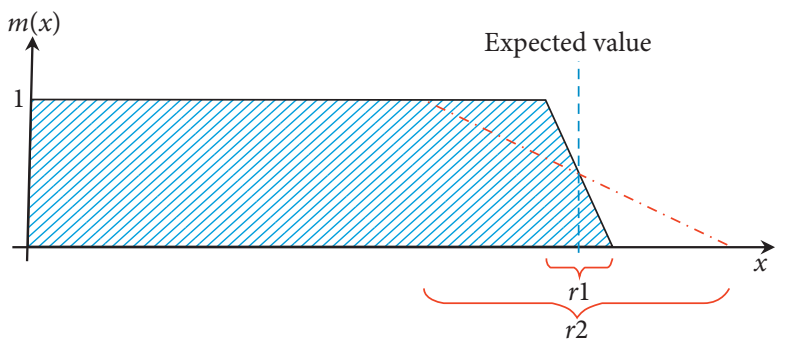

FIGURE 8: Impact of fuzzy parameter variations $(r)$ over the MFs.
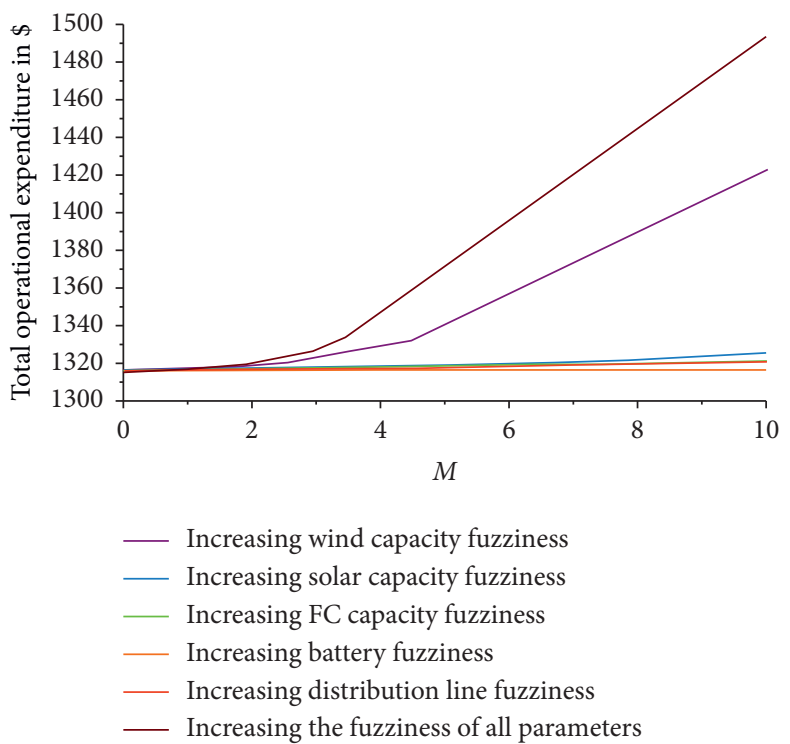

Figure 9: Impact of fuzzy parameter variations over the total operating cost.

has some other causes such as 7.3 percent increment in bought power from power market and 7.4 percent increment in generated power of the FC. All of the green power subsidies are eliminated in case 7 . Obtained results for this case are close to case 6 . There is 11 percent increase in case 7 compared to case 6 that is because of the elimination of solar energy subsidy.

The optimum daily programming of the MG for case 7 is captured in Figures 10 and 11 . In comparison to case 4, the generated power of the FC and battery activity are grown and decreased, respectively.

4.6. Variation Effects on the ESS and FC Capacities. Here, 4 cases are considered to study the effects of ESS and FC capacities. Compared to case 8 in Table 4, capacities of the FC and battery grow by 50 percent in case 9 and case 10 . In these two cases, the operational expenses reduce around 1.3 percent, while some small differences exist. Furthermore, 50 percent increment of FC capacity leads to increase its generated power up to 20 percent, decrease the purchased power from the main grid around 27 percent, and increase the sold power to the grid around 20 percent. Also, 50 percent growth in ESS capacity results in 6 and 7 percent 


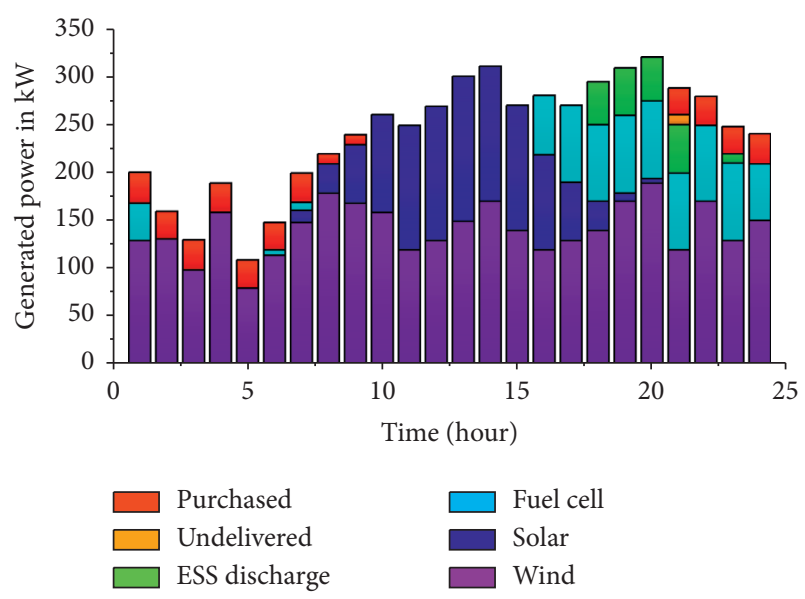

FIGURE 10: Optimum scheduling for various power sources and purchased power from the power market regardless of the fixed subsidies.

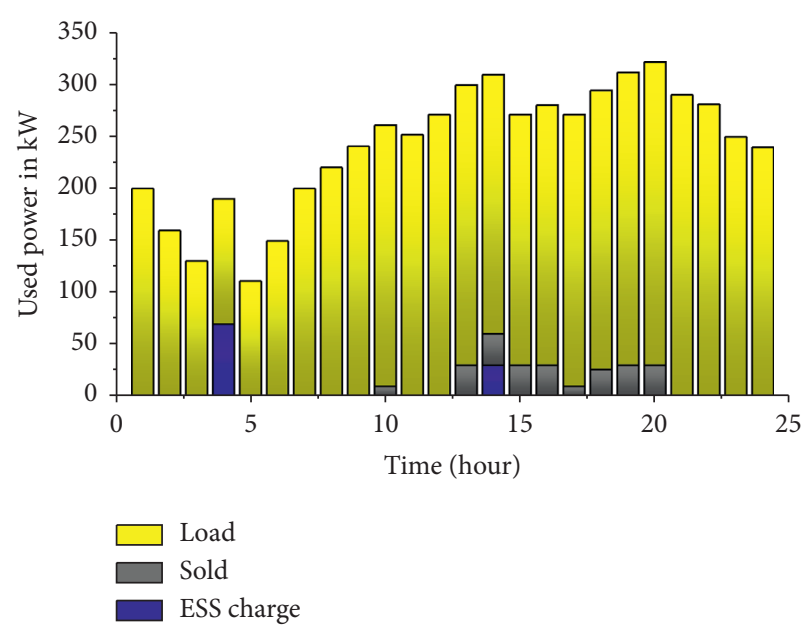

FIGURE 11: Optimum power consumption in the MG regardless of the fixed subsidies.

growth of ESS and FC activity, respectively. By comparison with case 9 and case 10, FC capacity growth results in a lower purchased power and more sold power from and to the grid.

Figure 12 depicts the obtained results of higher FC and battery capacity. According to this figure, when the FC capacity increases more than $100 \mathrm{~kW}$ and the battery capacity remains in a fixed amount $(200 \mathrm{~kW})$, total MG profit does not enhance. Besides, when the battery capacity raises more than $250 \mathrm{~kW}$ and the FC capacity remains in a fixed amount $(80 \mathrm{~kW})$, there is no improvement in the MG profit. Therefore, once the remaining factors of the MG are fixed, FC and battery capacities growth more than $100 \mathrm{~kW}$ and $250 \mathrm{~kW}$ does not have considerable impacts.

4.7. Effects of Distribution Line Capacity Variation. Growth of this capacity results in a higher operating flexibility to reduce the expenses of the MG. The obtained results of 3 various capacities are provided in Table 3 . According to these results, with growth of this capacity from $5 \mathrm{~kW}$ to

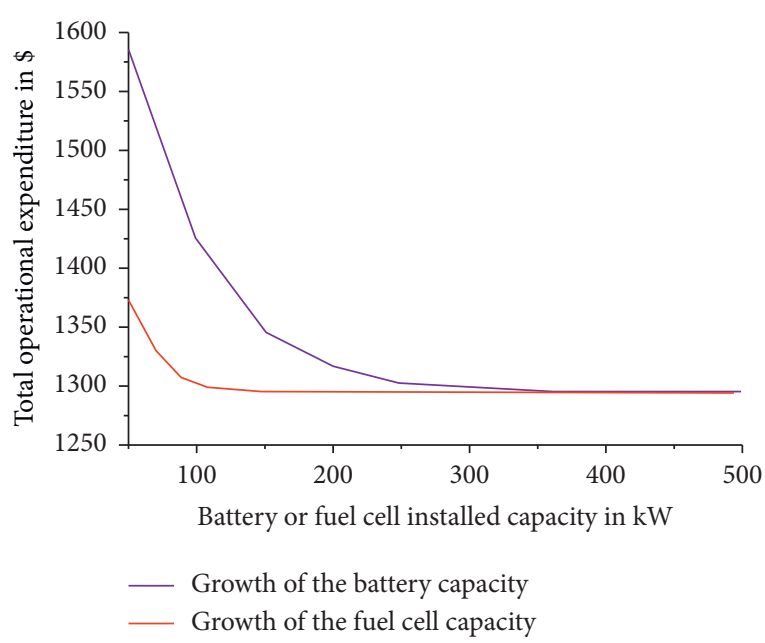

FIgURE 12: Total operating cost for various capacities of the fuel cell and ESS.

$60 \mathrm{~kW}$, activity of the battery reduces around 34 percent. Also, this increment results in growth of the exchanged power of the MG with the main grid around 650 percent. Moreover, increasing the distribution line capacity can reduce the sensitivity of the operating expenditure of the distribution capacity. This fact is visualized in Figure 13. According to this figure, the operating expenditure is constant for capacities more than $155 \mathrm{~kW}$. Thus, the best value of distribution capacity is $155 \mathrm{~kW}$ where other factors considered to be constant.

4.8. Modeling of the Load Fuzziness. From the practical aspect, we cannot predict the hourly amounts of the demanded power precisely. So, these values can be presented in the form of fuzzy sets. In this way, limitation (14) must be substituted with fuzzy limitations. Aiming this regard, we utilized 2 inequality conditions (as can be seen from Figure 14). In this figure, common region denotes the fuzziness of load in the vicinity of "a."

Therefore, equation (14) is substituted by 2 inequality limitations as follows:

$$
\begin{aligned}
& P_{w_{t}}+P_{p h_{t}}+P_{E S S_{t}}^{\mathrm{dc}}-P_{E S S_{t}}^{\mathrm{ch}}+P_{f c_{t}}-P_{\text {sell }_{t}}+P_{u_{t}} \leq P_{\text {load }_{t_{0}}}, \\
& \forall t \\
& \forall t \\
&-\left(P_{w_{t}}+P_{p h_{t}}+P_{E S S_{t}}^{\mathrm{dc}}-P_{E S S_{t}}^{\mathrm{ch}}+P_{f c_{t}}+P_{\text {sell }_{t}}+P_{\text {buy }_{t}}+P_{u_{t}}\right) \leq-P_{\text {load }_{t_{0}}}, \\
& \forall t \in T .
\end{aligned}
$$

Equally, limitations presented in equations (36), (45), and (55) can be rewritten in all 3 stated optimization problems. Also, it is considered that

$$
r_{\text {load }_{t}}=0.05 * P_{\text {load }_{t_{0}}} .
$$

Figures 15 and 16 illustrate the obtained results. In Figure 13, demanded power of each period is computed through subtraction of the total charged power of ESSs and sold power to the grid from the sum of the produced powers 


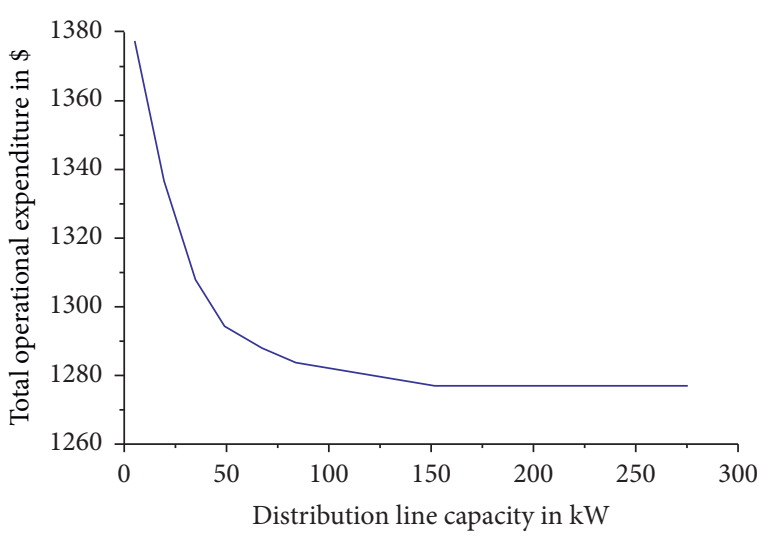

Figure 13: Total operating cost for various capacities of the transmission line.

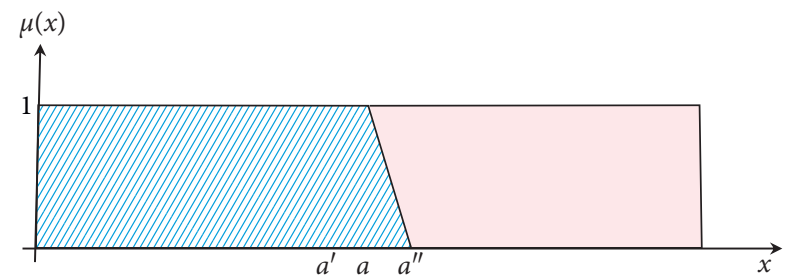

FIGURE 14: Graphical presentation of fuzzy equality limitations.

by the green power sources and the FC unit, purchased power from the grid and discharged power of the ESSs.

The demanded power in the base scenario in hour 7 is $195 \mathrm{~kW}$, whereas demanded power of equal time step is 190.23 kW in Figure 16. The reason behind this lower value is that the problem is aimed to obtain the lessen expenditure for load satisfying. So, the scheduling attempts to maximize the produced electricity from the cheapest power sources and reduce the variables, which raises the expenses. Demanded power has the highest importance in growth of the expenses. Hence, program attempts are considered to reduce the demanded power to $P_{\text {load }_{t_{0}}}-r_{\text {load }_{t}}$ (because demanded power $P_{\text {load }}-r_{\text {load }_{t}}$ can be converted to $P_{\text {load }_{t}}+r_{\text {load }}$ in the fuzzy form). Also, $\theta$ is utilized to control the load reduction. This parameter also can control the growth amount of the green power sources as well as the other fuzziness variables. The optimum amount of this parameter is obtained 0.4767 that can change the load to $P_{\text {load }_{t_{0}}}-\theta r_{\text {load }_{t}}=0.976 P_{\text {load }_{t_{0}}}$. Amount of the demanded power of the base scenario and its obtained amount by regarding the load fuzziness of the $7^{\text {th }}$ time interval are contrasted in Figure 17. As can be seen, the entire operational expenditure is reduced down to $1183 \$$, which is because of hourly demanded power reduction of the fuzzy scenario.

4.9. Additional Test Case. In this section, a new test case has been considered to show the efficiency of the suggested approach. The mentioned test case consists of $200 \mathrm{~kW}$ fuel

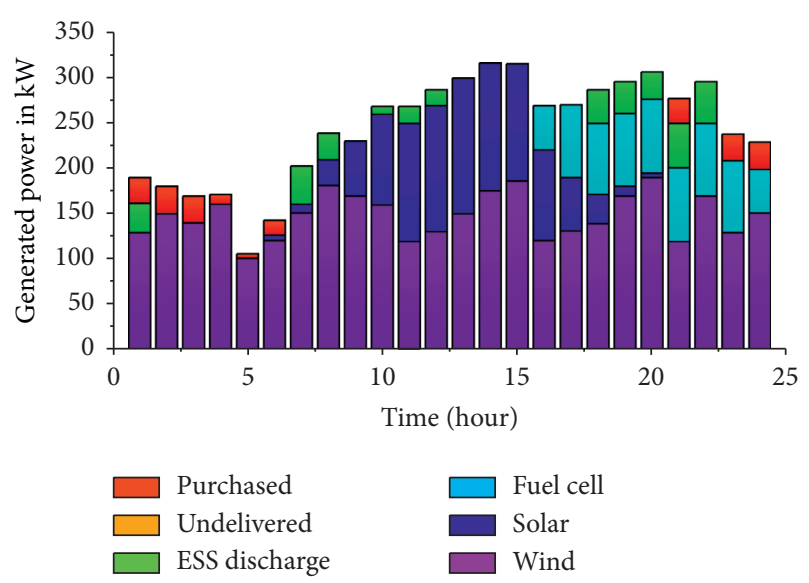

FIGURE 15: The optimum scheduling for various power sources and purchased power from the power market (considering the load uncertainty).

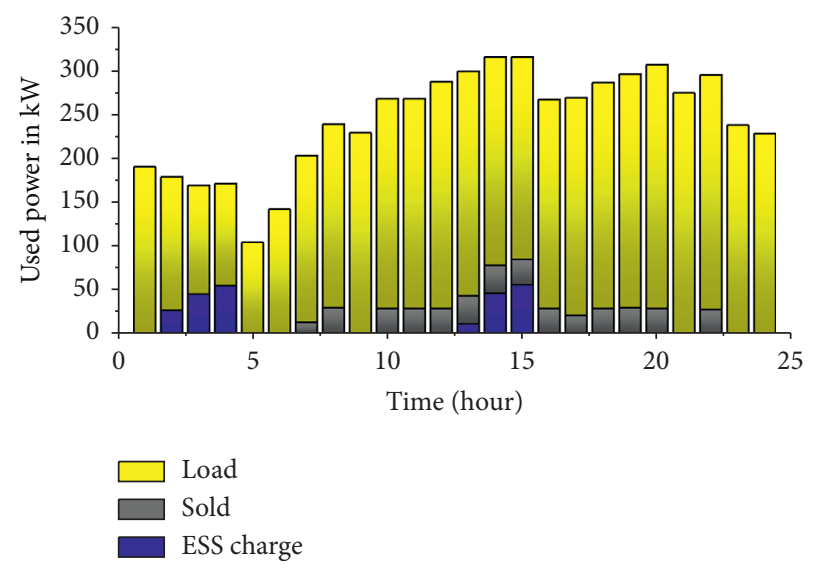

FIGURE 16: The optimum power consumption in the MG (considering the load uncertainty).

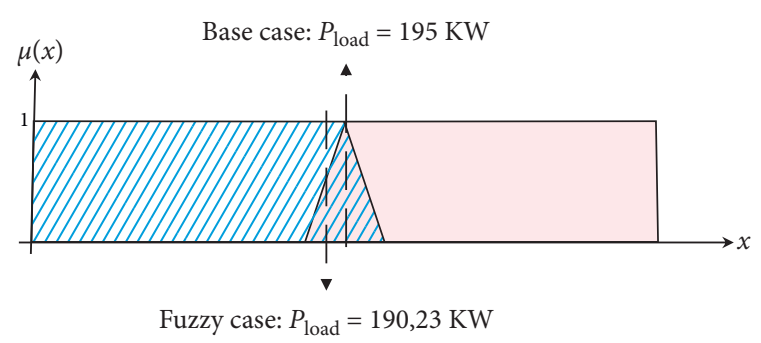

FIgURE 17: Comparison between the used power in the base scenario and the scenario considering the load fuzziness (at the $7^{\text {th }}$ time interval).

cells, a $120 \mathrm{~kW}$ saving system with a capacity of $480 \mathrm{kWH}$, and $360 \mathrm{~kW}$ wind power [27]. In this test case, a determined price of microgrids is applied for enhancing the markets for 15 to 38 hours in a day.

The mentioned test case is tested in 25th September, 2015 , while the hourly generation and consumption results 


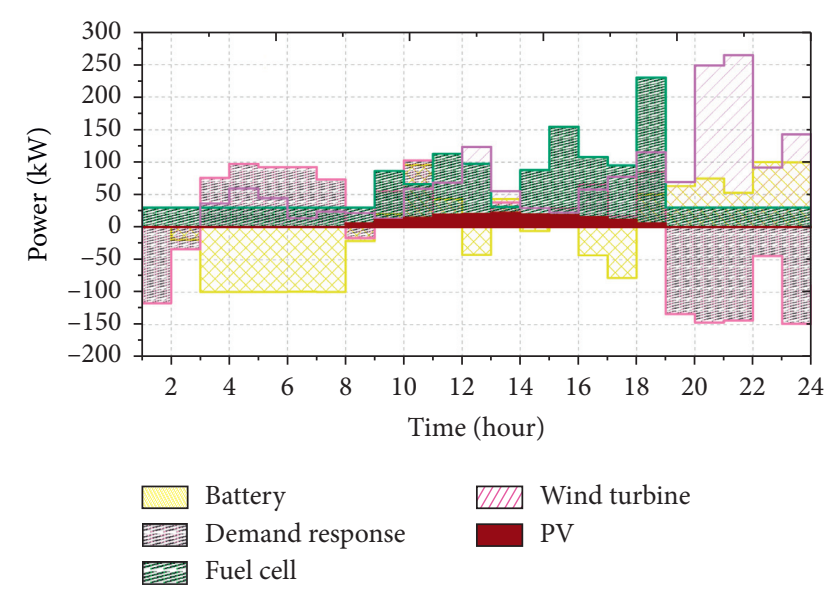

FIGURE 18: The value of produced power of each energy source on 25th September.

TABLE 5: Comparing various methods with the proposed test case for obtained cost.

\begin{tabular}{lcccc}
\hline Test day & $\begin{array}{c}\text { Support } \\
\text { vector } \\
\text { machine }\end{array}$ & $\begin{array}{c}\text { Support } \\
\text { vector } \\
\text { regression }\end{array}$ & $\begin{array}{c}\text { Mixed-integer } \\
\text { linear } \\
\text { programming }\end{array}$ & $\begin{array}{c}\text { Proposed } \\
\text { model }\end{array}$ \\
\hline $\begin{array}{l}25^{\text {th }} \\
\text { September }\end{array}$ & 205.16 & 215.36 & 216.66 & 233.44 \\
\hline
\end{tabular}

of microgrid components are presented in Figure 18. In this figure, the unbalanced power in photovoltaic and wind power was compensated with battery storage and demand response as well.

For more analysis, the maximum profit is evaluated. Obtained results are presented in Table 5. As shown in this table, the proposed approach could get better results in comparison with other models. Hence, the superiority of proposed approach is confirmed based on maximum profits by this model through comparison with other approaches.

\section{Conclusion}

In the present work, a novel method is proposed to optimally schedule the various power sources in a microgrid. It is aimed to minimize the total operating cost while preserving the comfort preferences of households. An MG is considered here containing WTs, PV systems, an FC, and battery, and it is connected to the main grid through a transmission line with bounded capacity. This paper also remarked various uncertain parameters such as solar irradiation and wind power, constraints of the FC and storage system energy production, capacity of the connection line, and uncertainty on the load. These uncertain parameters are implemented by the fuzzy concept. By application of the fuzzy concept, inequality limitations of the main model are substituted with fuzzy MFs considering fuzzy control factors $(r)$. This control parameter indicates the fuzziness level of the limitations. The suggested method is implemented on a practical test system for evaluation of its efficiency. Then, the optimum results are obtained and analyzed. Furthermore, effects of various factors are studied over the operating cost.

According to the obtained results, high uncertainty can result in growth of the total operating cost. Also, effects of various supporting procedures of the network operator are studied over the optimum scheduling of the MG. Based on the obtained results, utilization of the distribution line widely depends on various procedures. In addition, various capacities of the FC and storage system are studied, and the optimal capacities are determined. Effects of the transmission line variations are studied in this paper. Then, the best amount of this capacity is determined based on the obtained results.

\section{Data Availability}

No data were used to support this study.

\section{Conflicts of Interest}

The authors declare that they have no conflicts of interest.

\section{References}

[1] T. Cheng, M. Chen, Y. Wang, Bo Li, T. Chen, and R. Xu, "Adaptive robust method for dynamic economic emission dispatch incorporating renewable energy and energy storage," Complexity, vol. 2018, Article ID 2517987, 13 pages, 2018.

[2] R. Zacharia, A. M. Stephan, and J. R. Nair, "Nanomaterials for renewable energy storage: synthesis, characterization, and applications," Journal of Nanomaterials, vol. 2015, Article ID 308483, 13 pages, 2015.

[3] M. Deissenroth, M. Klein, K. Nienhaus, and M. Reeg, "Assessing the plurality of actors and policy interactions: agent-based modelling of renewable energy market integration," Complexity, vol. 2017, Article ID 7494313, 24 pages, 2017.

[4] J. Lin, F. Meng, R. Chen, and Q. Zhang, "Preference attitudebased method for ranking intuitionistic fuzzy numbers and its application in renewable energy selection," Complexity, vol. 2018, Article ID 6251384, 14 pages, 2018.

[5] J. Xiao, J. Cai, and X. Wang, "A hesitant fuzzy linguistic multicriteria decision-making method with interactive criteria and its application to renewable energy projects selection," Mathematical Problems in Engineering, vol. 2017, Article ID 9634725, 14 pages, 2017.

[6] L. Martínez-Villaseñor, H. Ponce, and A. Hernández, "Analysis of constraint-handling in metaheuristic approaches for the generation and transmission expansion planning problem with renewable energy," Complexity, vol. 2018, Article ID 1438196, 22 pages, 2018.

[7] J.-S. R. Jang, "ANFIS: adaptive-network-based fuzzy inference system," IEEE Transactions on Systems, Man, and Cybernetics, vol. 23, no. 3, pp. 665-685, 1993.

[8] H. Morais, P. Kádár, P. Faria, Z. A. Vale, and H. M. Khodr, "Optimal scheduling of a renewable micro-grid in an isolated load area using mixed-integer linear programming," Renewable Energy, vol. 35, no. 1, pp. 151-156, 2010.

[9] B. Zhao, Y. Shi, X. Dong, W. Luan, and J. Bornemann, "Shortterm operation scheduling in renewable-powered microgrids: a duality-based approach," IEEE Transactions on Sustainable Energy, vol. 5, no. 1, pp. 209-217, 2014. 
[10] M. Jadidbonab et al., "Short-term self-scheduling of virtual energy hub plant within thermal energy market," IEEE Transactions on Industrial Electronics, vol. 68, no. 4, 2020.

[11] H. R. Gholinejad, A. Loni, J. Adabi, and M. Marzband, "A hierarchical energy management system for multiple home energy hubs in neighborhood grids," Journal of Building Engineering, vol. 28, Article ID 101028, 2020.

[12] M. Nazari-Heris, M. A. Mirzaei, B. Mohammadi-Ivatloo, M. Marzband, and S. Asadi, "Economic-environmental effect of power to gas technology in coupled electricity and gas systems with price-responsive shiftable loads," Journal of Cleaner Production, vol. 244, p. 118769, 2020.

[13] M. Marzband, F. Azarinejadian, M. Savaghebi, E. Pouresmaeil, J. M. Guerrero, and G. Lightbody, "Smart transactive energy framework in grid-connected multiple home microgrids under independent and coalition operations," Renewable Energy, vol. 126, pp. 95-106, 2018.

[14] R. Das, Y. Wang, G. Putrus et al., "Multi-objective technoeconomic-environmental optimisation of electric vehicle for energy services," Applied Energy, vol. 257, p. 113965, 2020.

[15] M. A. Mirzaei, A. Sadeghi-Yazdankhah, B. MohammadiIvatloo, M. Marzband, M. Shafie-khah, and J. P. S. Catalão, "Integration of emerging resources in IGDT-based robust scheduling of combined power and natural gas systems considering flexible ramping products," Energy, vol. 189, p. 116195, 2019.

[16] G. Cau, D. Cocco, M. Petrollese, S. Knudsen Kær, and C. Milan, "Energy management strategy based on short-term generation scheduling for a renewable microgrid using a hydrogen storage system," Energy Conversion and Management, vol. 87, pp. 820-831, 2014.

[17] A. Malheiro, P. M. Castro, R. M. Lima, and A. Estanqueiro, "Integrated sizing and scheduling of wind/PV/diesel/battery isolated systems," Renewable Energy, vol. 83, pp. 646-657, 2015.

[18] R. Palma-Behnke, C. Benavides, F. Lanas et al., "A microgrid energy management system based on the rolling horizon strategy," IEEE Transactions on Smart Grid, vol. 4, no. 2, pp. 996-1006, 2013.

[19] M. Sedghi and M. Aliakbar-Golkar, "Optimal storage scheduling in distribution network considering fuzzy model of PEVs," in Proceedings of the Electrical Power Distribution Networks Conference, IEEE, Tehran, Iran, 2013.

[20] S. Fan, L. Piao, and Q. Ai, "Fuzzy day-ahead scheduling of virtual power plant with optimal confidence level," IET Generation, Transmission \& Distribution, vol. 10, no. 1, pp. 205-212, 2016.

[21] R.-H. Liang and J.-H. Liao, "A fuzzy-optimization approach for generation scheduling with wind and solar energy systems," IEEE Transactions on Power Systems, vol. 22, no. 4, pp. 1665-1674, 2007.

[22] S. Faddel, A. T. Al-Awami, and M. A. Abido, "Fuzzy optimization for the operation of electric vehicle parking lots," Electric Power Systems Research, vol. 145, pp. 166-174, 2017.

[23] A. Rezvani, M. Gandomkar, M. Izadbakhsh, and A. Ahmadi, "Environmental/economic scheduling of a micro-grid with renewable energy resources," Journal of Cleaner Production, vol. 87, pp. 216-226, 2015.

[24] M. Inuiguchi and J. Ramík, "Possibilistic linear programming: a brief review of fuzzy mathematical programming and a comparison with stochastic programming in portfolio selection problem," Fuzzy Sets and Systems, vol. 111, no. 1, pp. 3-28, 2000.
[25] M. Inuiguchi, "Stochastic programming problems versus fuzzy mathematical programming problems," Japanese Journal of Fuzzy Theory and Systems, vol. 4, no. 1, pp. 97-109, 1992.

[26] N. Jauuat and H. Whitehead, "Movement, distribution and feeding success of sperm whales in pacific ocean, over scales of days and tens of kilometers," Aquatic Mammals, vol. 25, no. 1, pp. 1-13, 1999.

[27] X. Lin, C. Chen, and S. Gauzily, "Integrated bidding strategy of distributed energy resources based on novel prediction and market model," International Journal of Energy Research, vol. 44, no. 5, pp. 4048-4062, 2020. 